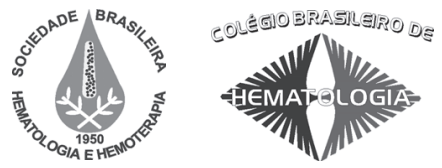

\title{
Mutações predisponentes à trombofilia em indivíduos de Minas Gerais - Brasil com suspeita clínica de trombose
}

\author{
Predisposing thrombophilic mutations in individuals with clinical suspicion of thrombosis from \\ Minas Gerais, Brazil
}

\author{
Sabrina P. Guimarães ${ }^{1}$ \\ Jerusa B. B. Soares ${ }^{2}$ \\ Vanessa C. Oliveira ${ }^{l}$ \\ Victor C. Pardini ${ }^{3}$ \\ Alessandro C. S. Ferreira ${ }^{4}$
}

\begin{abstract}
A trombose é reconhecidamente uma doença de caráter multifatorial. Sua ocorrência está intimamente relacionada à presença de fatores genéticos e adquiridos que concorrem isoladamente ou em associação para o seu desencadeamento. No entanto, a frequência dos fatores genéticos pode variar de acordo com a origem étnica e com outros aspectos epidemiológicos dos grupos de indivíduos e populações estudadas. No Brasil, dados referentes a individuos brasileiros e em especial do estado de Minas Gerais são escassos. O objetivo do presente estudo foi investigar a frequência das mutações fator V Leiden e G20210A no gene protrombina em 1.103 individuos com suspeita clínica de trombofilia, empregando a técnica da reação em cadeia da polimerase seguida de restrição enzimática (PCR-RFLP). Os dados foram analisados usando-se o programa Epi Info versão 6.04. A amostra consistiu de 76,16\% mulheres e 23,84\%

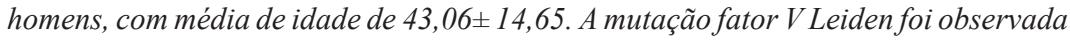
em heterozigose em 7,52\% dos individuos e em 0,36\% em homozigose. A mutação G20210A no gene da protrombina apresentou-se em heterozigose em 5,90\% dos indivíduos e em homozigose em $0,18 \%$. O presente trabalho mostra a importância dos testes genéticos conforme o perfil da população analisada, ressaltando informações epidemiológicas da população brasileira e beneficios clínicos. Rev. Bras. Hematol. Hemoter. 2009;31(1):19-24.
\end{abstract}

Palavras-chave: Trombofilia; fator V Leiden; mutação no gene da protrombina; Brasil.

\section{Introdução}

A predisposição elevada ao tromboembolismo devido à presença de fatores genéticos ou adquiridos é denominada trombofilia. ${ }^{1}$ A trombose é uma doença de caráter multifatorial, e tanto fatores de risco genéticos quanto adquiridos podem estar presentes em um mesmo indivíduo, sendo o desenvolvimento do tromboembolismo resultante da interação sinérgica ou não entre esses fatores. Pacientes com trombofilia hereditária exibem uma predisposição aumentada à trombose recorrente e o evento trombótico ocorre, em geral, antes dos 45 anos de idade. ${ }^{2}$ A morbimortalidade da trombose venosa e de suas complicações constitui hoje um importante problema de saúde pública. Nos Estados Unidos, foi estimada uma mortalidade anual por tromboembolismo pulmonar de 50 mil pessoas e a ocorrência de 300 mil e 600 mil

\footnotetext{
${ }^{1}$ Farmacêutica-bioquímica.

${ }^{2}$ Bióloga.

${ }^{3}$ Diretor do Instituto Hermes Pardini - Belo Horizonte, MG.

${ }^{4}$ Gerente de Inovação e Procedimentos Especiais do Instituto Hermes Pardini - Belo Horizonte, MG.

Departamento de Genética Humana - Instituto Hermes Pardini - Belo Horizonte, MG.

Correspondência: Alessandro Clayton de Souza Ferreira

Departamento de Genética Humana - Instituto Hermes Pardini

Rua Maranhão, 1040 - Funcionários

30150-330 - Belo Horizonte, MG - Brasil

Fax.: (+55 31) 3228-6524

Email: alessandro@labhpardini.com.br
} 
internações por trombose venosa profunda e tromboembolismo pulmonar. Castro-Silva, a partir de dados de internações do SUS, estimou no Brasil cerca de $28 \mathrm{mil}$ hospitalizações por embolia pulmonar com 4.247 mortes por ano. ${ }^{3}$ Em contraste com as doenças monogênicas, onde mutações em um único gene resultam na doença, em doenças multigênicas, como a trombose venosa, diferentes mutações em genes distintos interagem para ocasionar o evento. Desta forma, a predisposição à trombose venosa associada com cada alteração genética isolada é relativamente baixa, porém a presença de mutações em vários genes aumenta significativamente o risco de desenvolvimento da doença. $^{2}$

As trombofilias hereditárias são, em geral, decorrentes de mutações nos genes que codificam fatores da coagulação, como a mutação G1691A no gene do fator V e a mutação G20210A no gene da protrombina ou de alterações ligadas aos inibidores fisiológicos da coagulação (antitrombina, proteína C e proteína S). A mutação fator V Leiden (G1691A) resulta no fenótipo chamado resistência à proteína $\mathrm{C}$ ativada e é observada em $20 \%$ a $40 \%$ dos pacientes com trombose. ${ }^{4}$ Já a mutação G20210A no gene da protrombina pode acarretar elevação dos níveis plasmáticos de protrombina, da ordem de $30 \%$ (hiperprotrombinemia) e consequente coagulação exacerbada, levando ao aumento do risco de trombose. ${ }^{5,6}$

A mutação fator $\mathrm{V}$ Leiden está presente em diversas populações caucasianas, sendo que a prevalência de heterozigose na população varia de $2 \%$ a $13 \%$ e é extremamente rara entre africanos, chineses, japoneses, ameríndios e povos do sul da Ásia. ${ }^{7}$ A mutação G20210A no gene da protrombina é encontrada em $2 \%$ a $4 \%$ dos caucasianos e, em pacientes que já sofreram trombose, a frequência é de aproximadamente 20\%. ${ }^{8,9}$ A mutação G20210A no gene da protrombina, assim como a mutação fator $\mathrm{V}$ Leiden, é extremamente rara em populações não caucasianas. ${ }^{8}$

Sabe-se que a frequência desses fatores pode variar entre diferentes populações ou mesmo dentro de uma grande população como a brasileira, altamente miscigenada e que sofreu, em sua formação em diferentes regiões do país, a contribuição de diferentes grupos étnicos, justificando análises destes fatores em indivíduos de diferentes regiões do país. ${ }^{10}$ Neste sentido, o objetivo do atual estudo é investigar a frequência dos fatores trombofílicos, genéticos, fator $\mathrm{V}$ Leiden e mutação no gene da protrombina em indivíduos com suspeita clínica de tromboembolismo no estado de Minas Gerais, Brasil.

\section{Casuística e Método}

O estudo resulta de uma análise retrospectiva de 1.103 indivíduos com suspeita clínica de trombofilia submetidos ao estudo molecular das mutações fator V Leiden (FVL) e G20210A, no período de 2004 a 2007, no Instituto Hermes
Pardini, Departamento de Genética Humana, Belo Horizonte, Minas Gerais, Brasil. Neste estudo, apenas foram incluídos indivíduos cujo pedido médico para análise das mutações FVL e G20210A constasse como indicação clínica à suspeita de trombofilia. Os indivíduos envolvidos no estudo se apresentaram numa faixa etária de 10 a 89 anos, com média de idade correspondente a 43,06 $\pm 14,65$ anos. A amostra foi constituída por 263 homens e 840 mulheres. Idade, sexo e genótipos para as mutações FVL e G20210A foram analisados. O estudo constitui um levantamento dos resultados de exames de trombofilias, com finalidade epidemiológica e estatística, de acordo com os procedimentos da Comissão Nacional de Ética em Pesquisa (Conep).

Amostras de $5 \mathrm{~mL}$ de sangue coletado em EDTA, por punção venosa em tubos a vácuo com sistema Vacutainer (Becton-Dickinson) foram usadas para a extração de DNA em FTA Card $\AA$, de acordo com as instruções do fabricante (Whatman England FTA ${ }^{\circledR}$ Classic). O critério etnia não foi considerado, uma vez que a população brasileira é uma das mais heterogêneas do mundo, o que impossibilita a definição precisa da origem étnica dos indivíduos baseando-se apenas em parâmetros morfológicos. ${ }^{10}$

As mutações foram investigadas pela técnica de PCRRFLP usando-se os oligonucleotídeos e endonucleases de restrição, como previamente descritos. ${ }^{11}$ As reações foram realizadas em termociclador Master Cycler Gradient ${ }^{\circledR}$. Os produtos de PCR foram submetidos à digestão com endonucleases de restrição por $4 \mathrm{~h}$ a $37^{\circ} \mathrm{C}$ com Mni I e Hind III (Invitrogen ${ }^{\circledR}$ ) para detecção das mutações G1691A e G20210A, respectivamente. As amostras foram analisadas em gel de agarose e coradas com Syber Green ${ }^{\circledR}$ (Invitrogen ${ }^{\circledR}$ ).

As análises estatísticas foram realizadas usando-se o programa Epi Info Version 6.04. ${ }^{12}$ Os dados foram comparados às frequências das mutações observadas no grupocontrole constituído de 324 indivíduos hígidos e não relacionados, de Minas Gerais, previamente formado no estudo de Sabino et al..$^{13}$ Odds ratio (ORs) foram usadas como medida de associação entre mutação e tromboembolismo e foram interpretadas como risco de desenvolvimento da doença. Níveis de significância foram estimados pelo teste qui-quadrado. Diferenças foram consideradas significativas quando $\mathrm{p}<0.05$.

\section{Resultados}

O presente estudo avaliou 1.103 indivíduos, com suspeita clínica de trombofilia, que foram submetidos à investigação genética das mutações FVL e G20210A. As características dos indivíduos participantes estão listadas na Tabela 1. A maioria dos indivíduos era do sexo feminino - 840 $(76,16 \%)$. Os participantes apresentaram uma média de idade de 43,06 $\pm 14,65$ anos. Não foi observada nenhuma diferença significativa em relação à idade entre homens e mulheres. 
Tabela 1. Caracterização dos indivíduos segundo sexo, média de idade e faixa etária (características gerais)

\begin{tabular}{|c|c|c|c|}
\hline População & $N(\%)$ & Média idade DP & Faixa etária (anos) \\
\hline Homens & $263(23,84 \%)$ & $45,66 \pm 14,65$ & $12-89$ \\
\hline Mulheres & $840(76,16 \%)$ & $40,46 \pm 14,69$ & $10-89$ \\
\hline Total & 1.103 & $43,06 \pm 14,65$ & $10-89$ \\
\hline
\end{tabular}

DP: desvio padrão

Tabela 2. Frequências genotípicas das mutações fator $V$ Leiden e G20210A no gene da protrombina

\begin{tabular}{cccc}
\hline Genótipos & Homens $(\%)$ & Mulheres $(\%)$ & $\mathrm{n}(\%)$ \\
\hline FVL & & & \\
GA & $22(8,37)$ & $61(7,26)$ & $83(7,52)$ \\
AA & - & $4(0,48)$ & $4(0,36)$ \\
GG & $241(91,63)$ & $775(92,26)$ & $1.016(92,12)$ \\
G20210A & & & \\
GA & $19(7,22)$ & $46(5,48)$ & $65(5,90)$ \\
AA & - & $2(0,24)$ & $2(0,18)$ \\
GG & $244(92,78)$ & $792(94,28)$ & $1.036(93,92)$ \\
\hline
\end{tabular}

$\mathrm{FVL}=$ Mutação no gene do fator $\mathrm{V}$

As frequências genotípicas para cada mutação estão listadas na Tabela 2. A mutação G1691A foi detectada em heterozigose em $83(7,52 \%)$ indivíduos e em homozigose em $4(0,36 \%)$. Entre os homens, $22(8,37 \%)$ eram heterozigotos para essa mutação, e, entre as mulheres, $61(7,26 \%)$. Apenas as mulheres apresentaram essa mutação em homozigose, correspondendo a quatro $(0,48 \%)$. A mutação G20210A foi detectada em heterozigose em $65(5,90 \%)$ indivíduos, sendo $19(7,22 \%)$ homens e $46(5,48 \%)$ mulheres. Em homozigose, foram observadas duas $(0,24 \%)$ mulheres. Na população estudada não houve diferença significativa para as frequências das mutações entre homens e mulheres.

Os indivíduos foram divididos em cinco faixas etárias ( 0 a 20, 21 a 30, 31 a 40, 41 a 50 anos e maiores de 50 anos). A maioria dos indivíduos do sexo masculino $(66,53 \%)$ estava concentrada na faixa acima de 40 anos, enquanto a maioria das mulheres $(58,21 \%)$ estava na faixa de 21 a 40 anos. Foram observados $91(34,59 \%)$ homens e $196(22,15 \%)$ mulheres acima de 50 anos, o que representa $56,74 \%$ do total de indivíduos avaliados. Cumpre mencionar que a maioria dos homens portadores das mutações predisponentes à trombofilia apresentava-se na faixa etária acima de 40 anos, enquanto a maior porcentagem de mulheres com as mutações em questão apresentava-se entre 31 e 40 anos. Observou-se uma frequência aumentada da mutação G20210A em heterozigose entre os homens quando comparada com a frequência entre as mulheres. Foram observados 43 (3,90\%) indivíduos menores de 20 anos com suspeita clínica de trombofilia.

As frequências das mutações avaliadas foram comparadas com frequências do estudo anteriormente realizado no estado de Minas Gerais. Houve diferença significativa para a mutação fator V Leiden $(\mathrm{OR}=6,51$; IC $95 \%=2,46-17,21$; $\mathrm{p}<0,001)$ em relação a indivíduos saudáveis sem suspeita clínica e histórico para TEV. Não houve diferença para a mutação G20210A no gene da protrombina $(\mathrm{OR}=1,78$; IC $95 \%=0,94-3,38 ; p=0,105)$. Foi também observado um grupo de indivíduos portadores das mutações G1691A e G20210A, correspondente a $11(1,0 \%)$ casos. Entre esses, oito $(72,7 \%)$ apresentavam menos de 50 anos de idade.

\section{Discussão}

Apesar da frequência elevada dos eventos trombóticos, suas causas não foram ainda completamente esclarecidas, e os fatores de risco não são totalmente conhecidos em indivíduos jovens da nossa população. A trombose é reconhecidamente uma doença de caráter multifatorial. Sua ocorrência está intimamente relacionada à presença de fatores genéticos e adquiridos que concorrem isoladamente ou em associação para o seu desencadeamento. Embora vários estudos tenham investigado fatores de risco genéticos e adquiridos em diversas populações, dados referentes a indivíduos brasileiros e em especial do estado de Minas Gerais são ainda escassos.

Em nosso meio, torna-se difícil e imprecisa a categorização dos indivíduos em relação à origem étnica, uma vez que os brasileiros formam uma das populações mais heterogêneas em todo o mundo, resultado de cinco séculos de cruzamentos inter-raciais entre pessoas de três continentes: colonizadores europeus, principalmente portugueses, escravos africanos e ameríndios nativos. Desde o século XVI, o Brasil tem recebido crescente número de imigrantes de várias partes do mundo, principalmente de portugueses e africanos, seguidos de italianos, espanhóis e alemães e, mais recentemente, de asiáticos. Durante esses cinco séculos, $58 \%$ dos imigrantes foram europeus, $40 \%$ africanos e $2 \%$ asiáticos. $^{10}$

Os fatores de risco para trombose arterial incluem, principalmente, a hipertensão arterial, o tabagismo, dislipidemias e o diabetes mellitus. ${ }^{9}$ Entre os fatores adquiridos clássicos que contribuem para a trombose venosa podemos citar a idade, o uso de certos medicamentos como os anticoncepcionais orais e a terapia de reposição hormonal, gravidez e puerpério, imobilização de membros ou de parte do corpo, traumas locais, câncer, presença de anticorpos antifosfolípides, cirurgias de grande porte, infecções e síndrome nefrótica. ${ }^{14-16}$ Embora a literatura separe os fatores de risco dos eventos trombóticos arteriais e venosos, estudos recentes apontam uma ligação entre eles, incluindo idade, obesidade, tabagismo, diabetes mellitus, hipertensão arterial e hiperlipidemia como fatores de risco comum. ${ }^{17}$ Além desses, fatores de risco genéticos como os polimorfismos FVL e G20210A representam fatores importantes associados à trombose. No entanto, a frequência desses fatores pode variar de acordo com a origem étnica e com outros 
aspectos epidemiológicos dos grupos de indivíduos e populações estudadas. Além da composição étnica da população avaliada, sabe-se que a forma como são selecionados os indivíduos para o estudo pode afetar significativamente os resultados. ${ }^{18}$

O fato de a maioria dos homens com suspeita de trombofilia se apresentar na faixa etária acima de 40 anos e da maior porcentagem de mulheres entre 21 e 40 anos pode indicar a relação entre os fatores ambientais e as faixas etárias de maior exposição aos fatores de risco adquiridos com a incidência de tromboembolismo, ${ }^{19} \mathrm{o}$ que não pode ser comprovado neste estudo, devido ao desconhecimento do diagnóstico conclusivo e/ou histórico dos indivíduos analisados.

Os dados observados estão de acordo com Silverstein et al., que encontraram uma incidência ligeiramente aumentada das mutações FVL e G20210A em mulheres jovens e uma discreta concentração destas referidas mutações em homens mais velhos. ${ }^{19}$ Embora fatores de risco específicos tais como o uso de contraceptivos e a terapia de reposição hormonal estejam associados ao tromboembolismo venoso (TEV), estudos mostraram que não há uma diferença significativa na incidência de TEV entre homens e mulheres. ${ }^{20,21}$ No presente estudo também não foi observada diferença significativa para as frequências das mutações entre homens e mulheres. Cushman et al..$^{22}$ relataram incidência similar entre homens e mulheres, exceto um aumento em torno de duas vezes em homens com idade acima de 75 anos.

$\mathrm{O}$ presente estudo indica a mutação fator $\mathrm{V}$ Leiden como um importante fator de risco entre indivíduos com suspeita clínica de trombofilia $(\mathrm{OR}=6,51$; IC $95 \%=2,46-17,21$; $\mathrm{p}=0.000) .{ }^{13}$ As frequências observadas para as mutações FVL e G20210A estão de acordo com estudos prévios realizados no estado de Minas Gerais. ${ }^{13,23}$ Contudo, é importante ressaltar que a maioria dos estudos de associação entre as frequências das mutações predisponentes à trombofilia apresenta um desenho caso-controle, onde são analisados pacientes diagnosticados para TEV e indivíduos hígidos como controles. O estudo constitui um levantamento das frequências dessas mutações entre indivíduos com suspeita clínica, o que corrobora com outros estudos, de forma a traçar um perfil para população de Minas Gerais, bem como avaliar se os critérios de investigação de trombofilia estão sendo bem empregados. Pode-se inferir que os indivíduos com suspeita clínica foram bem caracterizados, já que as frequências para as mutações predisponentes à trombofilia foram similares às frequências encontradas para pacientes de Minas Gerais.

Foram também observados $11(1,0 \%)$ indivíduos portadores das duas mutações em questão, o que sugere uma possível interação sinérgica dessas, bem como de outros fatores contribuindo para o risco de tromboembolismo. A existência de mais de uma alteração pró-trombótica não somente aumenta o risco de desenvolvimento de eventos trombóticos, como também diminui a idade média de ocorrência do primeiro evento. ${ }^{24} \mathrm{Em}$ algumas famílias com casos de trombofilia, indivíduos com dois fatores genéticos desenvolvem o evento trombótico com maior frequência e mais cedo quando comparados com aqueles que possuem apenas um fator. ${ }^{14}$ Os indivíduos portadores de FVL e G20210A no gene da protrombina simultaneamente têm o risco de trombose venosa recorrente aumentado em 3,7 vezes quando comparados a indivíduos que são somente heterozigotos para FVL..$^{25,26}$ Contudo, existem indivíduos portadores de mutações de importância em trombofilia, tais como o FVL e G20210A no gene da protrombina, que podem permanecer assintomáticos por toda a vida, sugerindo a existência de outros fatores. ${ }^{27}$

Observa-se no estudo uma frequência aumentada de mulheres em relação aos homens. Para mulheres com fator genético predisponente conhecido e sem história familiar de TEV, os polimorfismos FVL e G20210A aumentam o risco em três a quatro vezes para TEV, enquanto a deficiência de AT, PC e OS aumenta o risco em oito a dez vezes. ${ }^{28}$

Sabe-se que, com o aumento da idade, eventos trombóticos tendem a ocorrer com maior frequência, devido às mudanças que o próprio organismo sofre, incluindo o sistema hemotástico e as alterações nos hábitos de vida. ${ }^{3}$ Estudos mostram que a incidência de tromboembolismo venoso aumenta espontaneamente com a idade. ${ }^{19,20}$ Assim, há uma variação grande na incidência de trombose de acordo com a faixa etária e com a população estudada, bem como há variação em relação à recorrência do evento trombótico. ${ }^{29-31}$ Pacientes com idade acima de 40 anos têm um risco significativamente aumentado quando comparados a pacientes jovens e esse risco praticamente dobra a cada década subsequente. ${ }^{32}$ A ocorrência de trombose constitui um evento raro em crianças e jovens. No presente estudo também foram observados indivíduos menores de 20 anos com suspeita clínica de trombofilia. Em geral, quando a trombose ocorre em crianças, há uma conjugação de fatores de risco variados, tanto genéticos quanto adquiridos. De acordo com Rosendaal et al., ${ }^{8}$ não é apenas raro observar trombose em crianças, mas aquelas que manifestam a doença apresentam de três a quatro fatores de risco associados.

\section{Conclusão}

O direcionamento clínico de pacientes com TEV ou indivíduos com suspeita clínica de trombofilia envolve uma avaliação complexa de fatores de risco adquiridos e genéti$\cos$, visto que a trombose representa uma doença multifatorial. Embora o presente estudo avalie uma população com suspeita clínica sem diagnóstico confirmado para trombofilia, ele representa um dado epidemiológico importante para o estado de Minas Gerais, já que foi avaliado um número significativo de indivíduos. Além disso, as frequências das principais mutações predisponentes à trom- 
bofilia confirmaram resultados encontrados em pacientes com TEV diagnosticado de estudo anterior realizado em Minas Gerais, ${ }^{13}$ o que permite afirmar que a investigação clínica está compatível com o diagnóstico.

O Brasil constitui uma das populações mais heterogêneas do mundo, o que se reflete nos parâmetros genéticos das diversas doenças hereditárias, trazendo diversidade gênica para a população e, junto, a necessidade de estudos bem conduzidos que colaborem na elucidação da etiologia dessas doenças. Finalmente, deve-se considerar que, por serem doenças graves, de caráter hereditário, a identificação precoce destas mutações possibilita a identificação de portadores assintomáticos que poderão receber orientação adequada em situações de risco, visando evitar a ocorrência de eventos trombóticos.

\section{Abstract \\ Thrombosis is known to be a multifactorial disease. It incidence is directly related to the presence of genetic and acquired factors that concur separately or in association to its appearance. However, the frequency of genetic factors can vary according to ethnic background and with other epidemiological aspects of populations. Data from Brazilian individuals and especially those from the State of Minas Gerais are scarce. The present study aims at investigating the frequencies of the factor $v$ Leiden and the G20210G prothrombin gene mutations of 1103 individuals with clinical suspicion of thrombophilia employing the Polymerase Chain Reaction technique followed by enzymatic restriction (PCR-RFLP). The data were analyzed using the Epi Info computer program version 6.04. The sample constituted of $76.16 \%$ women and $23.84 \%$ men, with an average age of $43.06 \pm 14.65$ years. The factor V Leiden mutation was observed as heterozygosis in $7.5 \%$ of individuals and as homozygosis in $0.36 \%$. The G20210A prothrombin gene mutation appeared as heterozygosis in 5.90\% of the individuals and as homozygosis in $0.18 \%$. The present study shows the importance of genetic tests taking into account the profile of the studied population, and stressing the epidemiological information of the Brazilian population and the clinical benefits. Rev. Bras. Hematol. Hemoter. 2009;31(1):19-24.}

Key words: Thrombophilia; factor V Leiden; prothrombin gene mutation; Brazil.

\section{Referências Bibliográficas}

1. Rodak BF. Hematology: Clinical principles and applications, Cap. 42 pg 609-625, cap. 44, p. 645-679, 835 p, $2^{\text {nd }}$ edição, W.B. Saunders Company, 2002.

2. Franco RF. Trombofilias hereditárias. In: Simpósio Hemostasia e Trombose. Medicina, v.34, p. 248-257, Ribeirão Preto, 2001.

3. Zago MA, Falcão RP, Pasquini R. Hematologia: fundamentos e prática. São Paulo: Atheneu, 2001. 1081p.

4. Dahlbäck B. Blood coagulation. Lancet. 2000;355(9215):1627-32.

5. Poort SR, Rosendaal FR, Reitsma PH, Bertina RM. A common genetic variation in the 3 '-untranslated region of the prothrombin gene is associated with elevated plasma prothrombin levels and an increase in venous thrombosis. Blood. 1996;88(10):3698703.

6. Lorenzi TF, D’Amico E, Daniel MM, Silveira PAA, Buccheri V. Manual de Hematologia - Propedêutica e Clínica. Cap. 9 pag. 595608, $3^{\mathrm{a}}$ edição, Medsi Editora Médica-2003. 641 p.

7. Bertina RM. Factor V Leiden and other coagulation factor mutations affecting thrombotic risk. Clin Chem. 1997;43(9):1678-83.

8. Rosendaal FR, Doggen CJ, Zivelin A, Arruda VR, Aiach M, Siscovick DS, et al. Geographic distribution of the $20210 \mathrm{G}$ to A prothrombin variant. Thromb Haemost. 1998;79(4):706-8.

9. Franco RF, Reitsma PH. Genetic risk factors of venous thrombosis. Hum. Hum Genet. 2001;109(4):369-84.

10. Parra FC, Amado RC, Lambertucci JR, Rocha J, Antunes CM, Pena SD. Color and genomic ancestry in Brazilians. Proc Natl Acad Sci USA. 2003;100(1):177-82.

11. Huber S, McMaster KJ, Voelkerding KV. Analytical evaluation of primer engineered multiplex polymerase chain reaction-restriction fragment length polymorphism for detection of factor $\mathrm{V}$ Leiden and prothrombin G20210A. J Mol Diagn. 2000;2 (3):153-7.

12. Dean AG, Dean JA, Coulombier D. Epi Info, version 6.04: a word processing database and statistics program for epidemiology on microcomputers. Atlanta: Centre of Disease Control and Prevention, 1997.

13. de Paula Sabino A, Guimarães DA, Ribeiro DD, Paiva SG, Sant'Ana Dusse LM, das Graças Carvalho M, et al. Increased Factor V Leiden frequency is associated with venous thrombotic events among young Brazilian patients. J Thromb Thrombolysis. 2007;24(3): 261-6.

14. Bertina RM. Genetic approach to thrombophilia. Thromb Haemost. 2001;86(1):92-103.

15. Rosendaal FR, Helmerhorst FM, Vandenbroucke JP. Oral contraceptives, hormone replacement therapy and thrombosis. Thromb Haemost. 2001;86(1):112-23.

16. Rosendaal FR, Helmerhorst FM, Vandenbroucke JP. Female hormones and thrombosis. Arterioscler Thromb Vasc Biol. 2002; 22(2):201-10.

17. Prandoni P, Ghirarduzzi A, Prins MH, Pengo V, Davidson BL, Sørensen $\mathrm{H}$, et al. Venous thromboembolism and the risk of subsequent symptomatic atherosclerosis. J Thromb Haemost. 2006;4(9):1891-6.

18. Bogardus ST Jr, Concato J, Feinstein AR. Clinical epidemiological quality in molecular genetic research: the need for methodological standards. JAMA. 1999;281(20):1919-26.

19. Silverstein MD, Heit JA, Mohr DN, Petterson TM, O'Fallon WM, Melton LJ 3rd. Trends in the incidence of deep vein thrombosis and pulmonary embolism: a 25-year population-based study. Arch Intern Med. 1998;158(6):585-93.

20. Anderson FA Jr, Wheeler HB, Goldberg RJ, Hosmer DW, Patwardhan NA, Jovanovic B, et al. A population-based perspective of the hospital incidence and case-fatality rates of deep vein thrombosis and pulmonary embolism. The Worcester DVT Study. Arch Intern Med. 1991;151(5):933-8.

21. White RH. The epidemiology of venous thromboembolism. Circulation. 2003;107(23 Suppl 1):I4-8.

22. Cushman M, Tsai A, Heckbert SR, et al. Incidence rates, case fatality, and recurrence rates of deep vein thrombosis and pulmonary embolus: the longitudinal investigation of thromboembolism etiology (LITE). Thromb Haemost. Stuttgard. 2001; 86(1). Abstract.

23. Dusse LM, Carvalho MG, Bragança WF, Paiva SG, Godoi LC, Fernandes AP. Inherited thrombophilias and pre-eclampsia in 
Brazilian womem. Eur J Obstet Gynecol Reprod Biol. 2007;134 (1):20-3.

24. Gemmati D, Serino ML, Trivellato C, Fiorini S, Scapoli GL. C677T substitution in the methylenetetrahydrofolate reductase gene as a risk factor for venous thrombosis and arterial disease in selected patients. Haematologica. 1999;84(9):824-8.

25. De Stefano V, Martinelli I, Mannucci PM, Paciaroni K, Chiusolo $\mathrm{P}$, Casorelli I, et al. The risk of recurrent deep venous thrombosis among heterozygous carriers of both factor $\mathrm{V}$ Leiden and the G20210A prothrombin mutation. N Engl J Med. 1999;341(11): 801-6.

26. Bauer KA. Management of thrombophilia. J Thromb Haemost. 2003;1(7):1429-34.

27. Martinelli I. Risk factors in venous thromboembolism. Thromb Haemost. 2001;86(1):395-403.

28. Middeldorp S. Oral contraceptives and the risk of venous thromboembolism. Gend Med. 2005; 2 Suppl A:S3-9.

29. Nordström M, Lindblad B, Bergqvist D, Kjellström T. A prospective study of the incidence of deep-vein thrombosis within a defined urban population. J Intern Med. 1992;232(2):155-60.

30. Kniffin WD Jr, Baron JA, Barrett J, Birkmeyer JD, Anderson FA Jr. The epidemiology of diagnosed pulmonary embolism and deep venous thrombosis in the elderly. Arch Intern Med. 1994; 154 (8): 861-6.

31. Hansson PO, Welin L, Tibblin G, Eriksson H. Deep vein thrombosis and pulmonary embolism in the general population. 'The Study of Men Born in 1913'. Arch Intern Med. 1997;157 (15):1665-70.

32. Anderson FA Jr, Spencer FA. Risk factors for venous thromboembolism. Circulation. 2003;107(23 Suppl 1):19-16.

Avaliação: Editor e dois revisores externos

Conflito de interesse: não declarado

Recebido: $16 / 05 / 2008$

Aceito após modificações: 22/07/2008 